\title{
Ex-vivo Assessment of Coronary Artery Atherosclerosis by Magnetic Resonance Imaging: Correlation with Histopathology
}

\author{
Everli P. S. Gonçalves Gomes ${ }^{1}$, Carlos Eduardo Rochitte ${ }^{1}$, Clerio F. Azevedo ${ }^{2}$, Pedro A. Lemos ${ }^{1}$, \\ Paulo Sampaio Gutierrez ${ }^{1}$ and Luiz Antonio M. César ${ }^{1, *}$
}

\author{
${ }^{1}$ Heart Institute (InCor) University of São Paulo Medical School, São Paulo, Brazil; ${ }^{2}$ D'Or Institute for Research and \\ Education (IDOR), Rio de Janeiro, Brazil
}

\begin{abstract}
Introduction: In recent years, high-resolution magnetic resonance imaging (MRI) has emerged as a very promising technique for studying atherosclerotic disease in humans. Aim: In the present study we sought to determine whether MRI allowed for the morphological characterization of the coronary vessel wall and atherosclerotic plaques using histopathological assessment as the reference standard. Methods: The study population consisted of 13 patients who died of acute myocardial infarction and underwent autopsy. The proximal portions of the coronary arteries were excised and were evaluated both by MRI and by histopathology. For each arterial segment, the following parameters were calculated through manual planimetry: 1. total vessel area (TVA); 2. luminal area (LA) and 3. plaque area (PA). Results: A total of 207 coronary artery cross-sections were found to be suitable for analysis by both MRI and histopathology and were included in the final analyses. Both methods demonstrated moderate to good agreement for the quantification of TVA (mean difference $=2.4 \pm 2.4 \mathrm{~mm}^{2}, 95 \%$ limits of agreement from -2.4 to $+7.2 \mathrm{~mm}^{2}$; CCC $=0.69,95 \%$ CI from 0.63 to 0.75 ), $\mathrm{LA}$ (mean difference $=0.0 \pm 1.7 \mathrm{~mm}^{2}, 95 \%$ limits of agreement from -3.3 to $+3.3 \mathrm{~mm}^{2} ; \mathrm{CCC}=0.84,95 \% \mathrm{CI}$ from 0.80 to 0.88 ) and PA (mean difference $=2.4 \pm 2.4 \mathrm{~mm}^{2}, 95 \%$ limits of agreement from -2.3 to $+7.1 \mathrm{~mm}^{2} ; \mathrm{CCC}=0.64$, 95\% CI from 0.58 to 0.71). Conclusion: In this ex vivo experimental model we demonstrated good agreement between coronary artery morphometrical measurements obtained by high-resolution MRI and by histopathology.
\end{abstract}

Keywords: Atherosclerosis, coronary artery disease, coronary vessels, histopathology, magnetic resonance imaging.

\section{INTRODUCTION}

Currently, atherosclerosis and its thrombotic complications are still the major cause of morbidity and mortality worldwide [1]. Invasive coronary angiography (ICA) remains the gold standard for the identification of clinically significant coronary stenosis and still remains as the best imaging technique for the diagnosis of obstructive coronary artery disease (CAD) [2]. However, ICA is basically limited to the assessment of the arterial lumen and does not provide information regarding the arterial wall or the presence of subclinical atherosclerosis. Other invasive imaging modalities such as intravascular ultrasound (IVUS) and IVUSderived techniques allows for the assessment of arterial remodeling, plaque characteristics, and optical coherence tomography allows near-histological resolution of plaque surface, providing detailed anatomical characterization of atherosclerotic plaques [3]. Nevertheless, these invasive techniques are expensive, demonstrate limited availability and, most importantly, are associated with a non-negligible risk of complications. In fact, despite advances in our understanding of the pathophysiology, pathogenesis, and new therapeutic strategies, the absence of an adequate

*Address correspondence to this author at the Instituto do Coração (InCor) Setor de Ressonância Magnética Cardiovascular, Av. Dr. Enéas de Carvalho Aguiar, 44, Andar AB, Cerqueira César, São Paulo, SP, 05403-000, Brazil; Tel: 55-11-2661-5587; Fax: 55-11-2661-5587;

E-mail: dcllucesar@incor.usp.br non-invasive imaging tool for early detection limits both the prevention and treatment of patients with various degrees of atherosclerotic CAD.

In recent years, high-resolution magnetic resonance imaging (MRI) has emerged as a very promising technique for studying atherosclerotic disease in humans [4-6], both ex-vivo and in-vivo. The emergence of high-resolution, rapid imaging methods has enabled MRI to non-invasively image the fine internal structure of atherosclerotic artery walls. However, it is important to highlight that, so far, the use of MRI for this purpose has been validated mostly in models of carotid or aortic wall atherosclerotic disease [7-11]. Even though there are several well conducted studies evaluating MRI as a non-invasive tool for the assessment of atherosclerotic CAD [4, 12-14], further pre-clinical experimental validation and evaluation of specific MRI techniques are still needed. Therefore, in the present ex-vivo experimental model we sought to determine whether MRI allowed for the morphological characterization of the coronary vessel wall and atherosclerotic plaques using histopathological assessment as the reference standard.

\section{METHODS}

\section{Patients}

The study population consisted of 13 patients ( 7 males and 6 females; mean age $68 \pm 12$ years) who died of acute myocardial infarction and underwent autopsy. All patients 
had a high-risk profile for atherosclerotic CAD (77\% had hypertension, $69 \%$ had dyslipidemia, $49 \%$ were diabetic, $54 \%$ were smokers and $31 \%$ former smokers). Informed consent to perform the study was obtained from all families and the protocol was approved by the Institutional Review Board.

\section{Tissue Preparation}

The hearts were fixed with a $10 \%$ buffered formalin solution. In order avoid coronary artery collapse during chemical fixation, which could interfere with morphological measurements, we used a dedicated device that performed constant perfusion of the coronary arteries with the formalin solution at a pre-defined perfusion pressure of $80 \mathrm{mmHg}$ during $2 \mathrm{~h}$. After fixation, the proximal portions of the coronary arteries were excised including the surrounding epicardial fat. The excised segments included a portion of the aortic wall encompassing the coronary ostia, the left main coronary artery and at least $3 \mathrm{~cm}$ of the left anterior descendent artery (LAD), left circumflex artery (LCX) and right coronary artery (RCA) (Fig. 1A). Then the arterial segments were evaluated both by MRI and by histopathology.

\section{MR Imaging}

All MRI exams were performed on a $1.5 \mathrm{~T}$ scanner (Signa CV/I, GE Medical Systems, Waukesha, WI). For noninvasive high-resolution imaging, the surface coil for temporomandibular articulation was used for transmission and reception. Before imaging, all arteries were filled with commercial edible soy oil and then were fixed at bottom of a container also filed with soy oil. Localizer images encompassing the entire container were acquired in three orthogonal (axial, sagittal and coronal). Then, all coronary arteries were evaluated using four different 3-dimensional (3D) pulse sequences: 1. 3D fast gradient-echo (3DGRE); 2. 3D fast gradient-echo with fat saturation (FAT SAT 3DGRE); 3. 3D T1-weighted fast spin-echo with fat saturation (FAT SAT 3DFSET1); and 4. 3D T2-weighted fast spin-echo (3DFSET2) (Fig. 1B-D). All four 3D datasets were acquired at the exact same position. The imaging parameters used in the 4 different sequences are detailed in Table 1 .

All MR image analyses were performed using the dedicated software package Vitrea $2^{\circledR}$ (Vital Images, Minnetonka, MM) for reconstruction of curved MPR and short-axis views of the coronary arteries, which were matched to the histological cross-sectional coronary slices. For each arterial segment, the following parameters were calculated through manual planimetry: 1 . total vessel area (TVA); 2. lumen area (LA) and 3. plaque area (PA).

\section{Histopathology}

For histopathological assessment, each coronary artery segment was sectioned into sequential transversal $3 \mathrm{~mm}$ slices in order to best match the corresponding MRI slices. Then, for each $3 \mathrm{~mm}$-slice, three paraffin-embedded sections were stained with Verhoeff. Similar to MR image analyses, the following parameters were determined by quantitative video-morphometry using an automated image analysis system (Quantimet 520 Image Analysis System, Cambridge
Instruments, Cambridge, UK): 1. total vessel area (TVA); 2. lumen area (LA) and 3. plaque area (PA) (Fig. 1E).

\section{Statistical Analysis}

Wilcoxon signed-rank tests were used to compare continuous variables, which were expressed as mean \pm SD. Fisher's exact test was used to compare categorical variables. Lin's concordance correlation coefficient (CCC) and BlandAltman analysis were used to evaluate the agreement between MRI and histopathological coronary artery morphometric measurements [15]. Analyses were performed with Stata software, version 10.0 (StataCorp, College Station, Texas). All tests were 2-tailed and a $\mathrm{p}<0.05$ was considered significant.

\section{RESULTS}

A total of 207 coronary artery cross-sections were found to be suitable for analysis by both MRI and histopathology and were included into the final analyses. The images obtained by all four MRI pulse sequences underwent visual subjective assessment by 2 experienced investigators and those derived from the FAT SAT 3DFSET1 pulse sequence were selected for coronary artery morphometrical measurements. The mean values of TVA, LA and PA measured by MRI and histopathology are summarized in Table 2.

The measurements of TVA by both methods demonstrated moderate to good agreement when we considered all coronary segments (mean difference $=2.4 \pm 2.4 \mathrm{~mm}^{2}, 95 \%$ limits of agreement from -2.4 to $+7.2 \mathrm{~mm}^{2}$; $\mathrm{CCC}=0.69$, $95 \%$ confidence interval from 0.63 to 0.75 ) as well as when we considered each coronary artery separately: LAD (mean difference $=3.1 \pm 3.3 \mathrm{~mm}^{2}, 95 \%$ limits of agreement from 3.4 to $+9.6 \mathrm{~mm}^{2} ; \mathrm{CCC}=0.55,95 \%$ confidence interval from 0.40 to 0.71 ), $\mathrm{LCx}$ (mean difference $=2.5 \pm 2.0 \mathrm{~mm}^{2}, 95 \%$ limits of agreement from -1.4 to $+6.4 \mathrm{~mm}^{2}$; $\mathrm{CCC}=0.69$, $95 \%$ confidence interval from 0.58 to 0.79 ) and RCA (mean difference $=2.0 \pm 2.1 \mathrm{~mm}^{2}, 95 \%$ limits of agreement from 2.1 to $+6.2 \mathrm{~mm}^{2} ; \mathrm{CCC}=0.69,95 \%$ confidence interval from 0.60 to 0.78 ) (Table 3 and Fig. 2).

Regarding the measurements of LA both methods also demonstrated good agreement considering the assessment of all coronary segments (mean difference $=0.0 \pm 1.7 \mathrm{~mm}^{2}$, $95 \%$ limits of agreement from -3.3 to $+3.3 \mathrm{~mm}^{2}$; $\mathrm{CCC}=0.84,95 \%$ confidence interval from 0.80 to 0.88 ) as well as each coronary artery separately: LAD (mean difference $=0.7 \pm 2.3 \mathrm{~mm}^{2}, 95 \%$ limits of agreement from -3.9 to $+5.3 \mathrm{~mm}^{2} ; \mathrm{CCC}=0.78,95 \%$ confidence interval from 0.69 to 0.88 ), $\mathrm{LCx}$ (mean difference $=-0.7 \pm 1.2 \mathrm{~mm}^{2}, 95 \%$ limits of agreement from -3.1 to $+1.7 \mathrm{~mm}^{2}$; $\mathrm{CCC}=0.78,95 \%$ confidence interval from 0.70 to 0.87 ) and RCA (mean difference $=0.1 \pm 1.4 \mathrm{~mm}^{2}, 95 \%$ limits of agreement from -2.6 to $+2.8 \mathrm{~mm}^{2} ; \mathrm{CCC}=0.89,95 \%$ confidence interval from 0.86 to 0.93 ) (Table 3 and Fig. 3).

The agreement between the measurements of PA by both methods was moderate when we considered all coronary segments (mean difference $=2.4 \pm 2.4 \mathrm{~mm}^{2}, 95 \%$ limits of agreement from -2.3 to $+7.1 \mathrm{~mm}^{2}$; $\mathrm{CCC}=0.64,95 \%$ confidence interval from 0.58 to 0.71 ) as well as when we considered each coronary artery separately: LAD (mean difference 

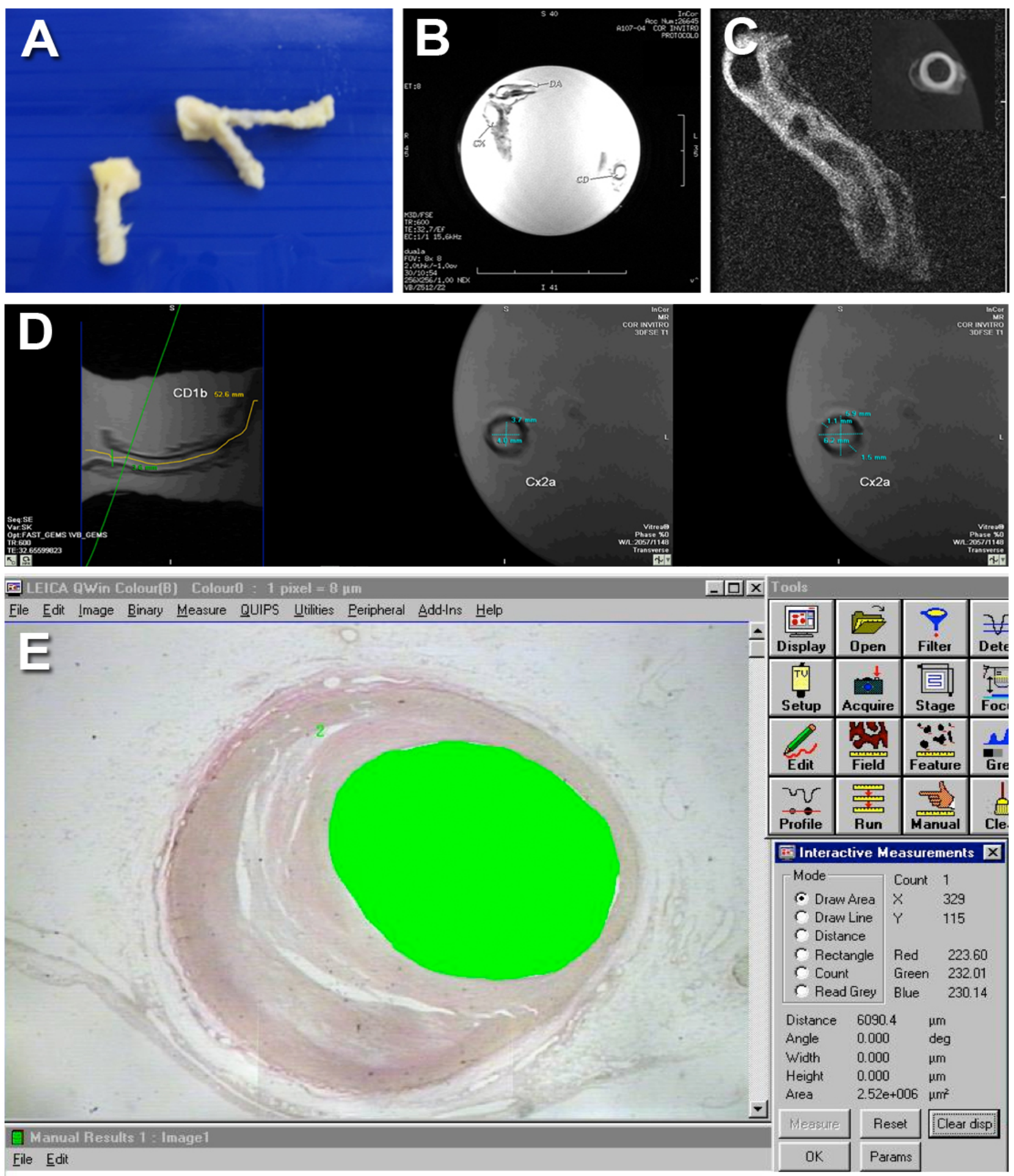

Fig. (1). A. Illustrative image demonstrating an example of excised coronary artery segments. B-D. Representative MR images: localizer image used to identify coronary arterial segments (B); High-resolution MR image acquired using the 3D fast gradient-echo with fat saturation pulse sequence (C); High-resolution MR images acquired using the 3D T1-weighted fast spin-echo with fat saturation (FAT SAT 3DFSET1) illustrating the quantitative assessment of coronary arterial segments (D). E. Illustrative image demonstrating histopathological assessment by quantitative video-morphometry using an automated image analysis system.

$=2.4 \pm 2.8 \mathrm{~mm}^{2}, 95 \%$ limits of agreement from -3.1 to +8.0 $\mathrm{mm}^{2} ; \mathrm{CCC}=0.65,95 \%$ confidence interval from 0.51 to 0.78 ), LCX (mean difference $=3.1 \pm 2.0 \mathrm{~mm}^{2}, 95 \%$ limits of agreement from -0.7 to $+7.0 \mathrm{~mm}^{2}$; $\mathrm{CCC}=0.48,95 \%$ confi- dence interval from 0.36 to 0.58 ) and RCA (mean difference $=1.9 \pm 2.3 \mathrm{~mm}^{2}, 95 \%$ limits of agreement from -2.6 to +6.4 $\mathrm{mm}^{2} ; \mathrm{CCC}=0.65,95 \%$ confidence interval from 0.55 to 0.75 ) (Table 3 and Fig. 4). 
Table 1. MR imaging parameters used in the 4 different pulse sequences used.

\begin{tabular}{|c|c|c|c|c|}
\hline TR (ms) & 40 & 40 & 600 & 2300 \\
\hline Flip angle $\left({ }^{\circ}\right)$ & 50 & 50 & 90 & 90 \\
\hline Matrix & $256 \times 160$ & $256 \times 256$ & $256 \times 256$ & $128 \times 128$ \\
\hline FOV $(\mathbf{c m})$ & 8 & 8 & 8 & 8 \\
\hline Slice thickness units? & 2 & 2 & 2 & 4 \\
\hline Number of slices & 34 & 34 & 34 & 18 \\
\hline
\end{tabular}

TR indicates repetition time; TE, echo time; FOV, field-of-view; 3DGRE, 3-dimensional fast gradient-echo; FAT SAT 3DGRE, 3-dimensional fast gradient-echo with fat saturation; FAT SAT 3DFSET1, 3-dimensional T1-weighted fast spin-echo with fat saturation; and 3DFSET2, 3-dimensional T2-weighted fast spin-echo.

Table 2. Coronary artery morphometrical measurements by MRI and by histopathology.

\begin{tabular}{|c|c|c|c|c|}
\hline & $\mathbf{N}$ & \multicolumn{2}{|c|}{ Median (Interquartile Range) } & $\mathbf{P}$ \\
\hline $\operatorname{TVA}\left(\mathbf{m m}^{2}\right)$ & & $14(11-17)$ & $12(9-15)$ & $<0.001$ \\
\hline $\mathbf{L A}\left(\mathbf{m m}^{2}\right)$ & & $4(2-6)$ & $4(3-6)$ & 0.44 \\
\hline LAD & 50 & & & \\
\hline $\operatorname{TVA}\left(\mathbf{m m}^{2}\right)$ & & $17(14-18)$ & $12(10-16)$ & $<0.001$ \\
\hline $\mathbf{L A}\left(\mathbf{m m}^{2}\right)$ & & $4(0-7)$ & $4(0-6)$ & 0.13 \\
\hline $\mathbf{P A}\left(\mathbf{m m}^{2}\right)$ & & $11(9-13)$ & $8(5-13)$ & $<0.001$ \\
\hline $\mathbf{L A}\left(\mathbf{m m}^{2}\right)$ & & $4(2-5)$ & $4(3-6)$ & $<0.001$ \\
\hline $\mathbf{P A}\left(\mathbf{m m}^{2}\right)$ & & $8(5-11)$ & $5(2-7)$ & $<0.001$ \\
\hline RCA & 94 & & & \\
\hline TVA $\left(\mathbf{m m}^{2}\right)$ & & $14(12-17)$ & $13(10-15)$ & $<0.001$ \\
\hline LA $\left(\mathbf{m m}^{2}\right)$ & & $5(3-7)$ & $5(3-7)$ & 0.28 \\
\hline $\mathbf{P A}\left(\mathbf{m m}^{2}\right)$ & & $10(7-11)$ & $7(5-10)$ & $<0.001$ \\
\hline
\end{tabular}

TVA indicates total vessel area; LA, lumen area; PA, plaque area; LAD, left anterior descending coronary artery; LCx, left circumflex coronary artery; RCA, right coronary artery; and MRI, magnetic resonance imaging.

\section{DISCUSSION}

In the present study we described a novel MRI technique for ex vivo coronary artery imaging and were able to demonstrate a moderate to good agreement between the morphometrical measurements obtained with this technique and those derived from specific histopathological analyses. Overall, the degree of agreement was found to be good for the quantification of LA and good to moderate for the assessment of TVA and PA. Importantly, the agreement between both methods was found to be similar for all 3 coronary arteries studied: LAD, LCx and RCA. 
Table 3. Assessment of agreement between MRI and histopathological measurements.

\begin{tabular}{|c|c|c|c|}
\hline & Mean difference $\left(\mathrm{mm}^{2}\right)$ & $95 \%$ Limits of agreement & CCC ( $95 \%$ confidence interval) \\
\hline \multicolumn{4}{|l|}{ All vessels } \\
\hline $\mathbf{L A}\left(\mathbf{m m}^{2}\right)$ & $0.0 \pm 1.7$ & -3.3 to +3.3 & $0.84(0.80$ to 0.88$)$ \\
\hline $\mathbf{P A}\left(\mathbf{m m}^{2}\right)$ & $2.4 \pm 2.4$ & -2.3 to +7.1 & $0.64(0.58$ to 0.71$)$ \\
\hline TVA $\left(\mathbf{m m}^{2}\right)$ & $3.1 \pm 3.3$ & -3.4 to +9.6 & $0.55(0.40$ to 0.71$)$ \\
\hline $\mathbf{L A}\left(\mathbf{m m}^{2}\right)$ & $0.7 \pm 2.3$ & -3.9 to +5.3 & $0.78(0.69$ to 0.88$)$ \\
\hline PA $\left(\mathbf{m m}^{2}\right)$ & $2.4 \pm 2.8$ & -3.1 to +8.0 & $0.65(0.51$ to 0.78$)$ \\
\hline \multicolumn{4}{|l|}{$\mathbf{L C x}$} \\
\hline PA $\left(\mathbf{m m}^{2}\right)$ & $3.1 \pm 2.0$ & -0.7 to +7.0 & $0.48(0.36$ to 0.58$)$ \\
\hline \multicolumn{4}{|l|}{ RCA } \\
\hline TVA $\left(\mathbf{m m}^{2}\right)$ & $2.0 \pm 2.1$ & -2.1 to +6.2 & $0.69(0.60$ to 0.78$)$ \\
\hline $\mathbf{L A}\left(\mathbf{m m}^{2}\right)$ & $0.1 \pm 1.4$ & -2.6 to +2.8 & $0.89(0.86$ to 0.93$)$ \\
\hline $\mathbf{P A}\left(\mathbf{m m}^{2}\right)$ & $1.9 \pm 2.3$ & -2.6 to +6.4 & $0.65(0.55$ to 0.75$)$ \\
\hline
\end{tabular}

TVA indicates total vessel area; LA, lumen area; PA, plaque area; LDA, left anterior descending coronary artery; LCx, left circumflex coronary artery; RCA, right coronary artery; and CCC, concordance correlation coefficient.

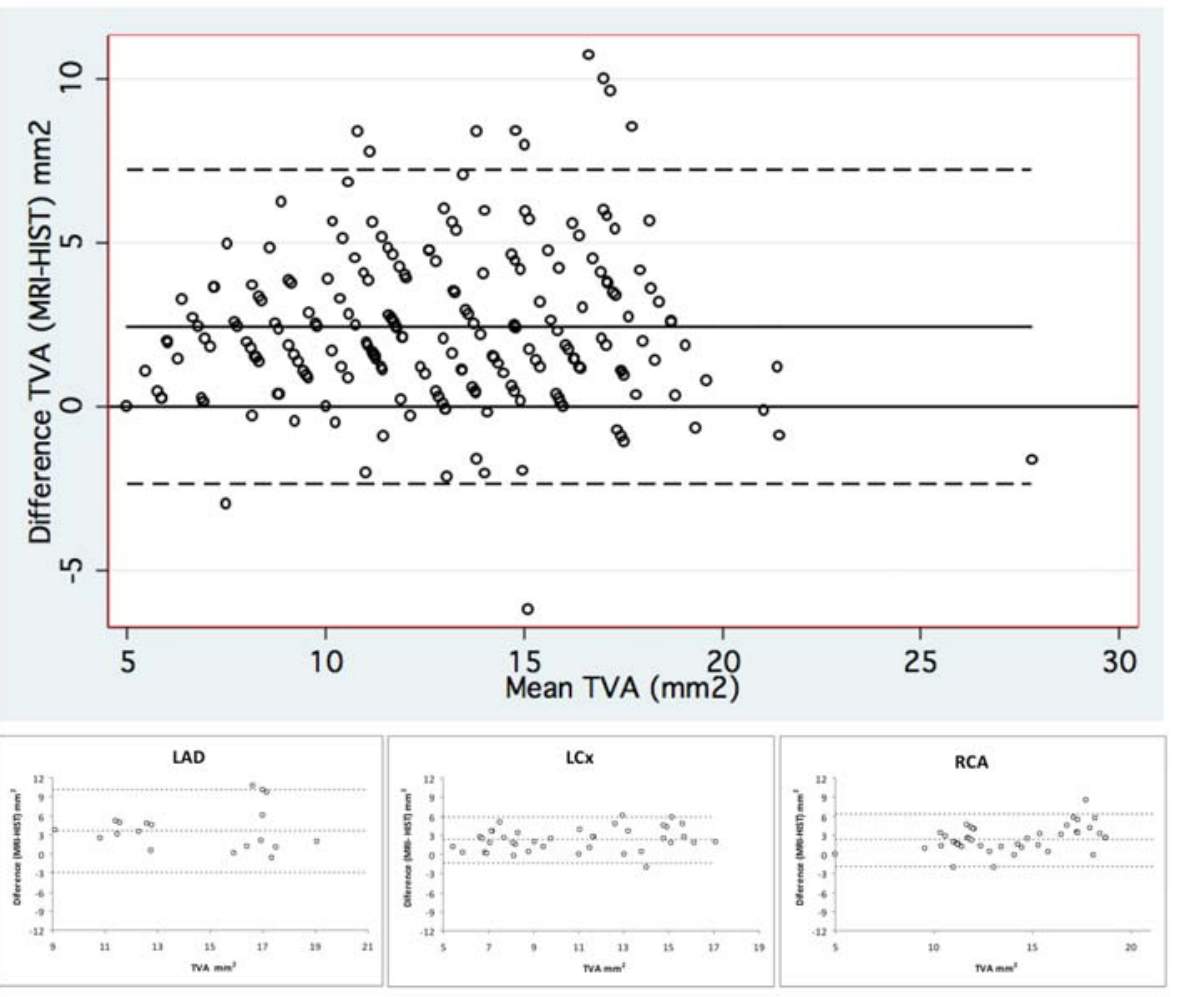

Fig. (2). Bland-Altman graphs illustrating the agreement between total vessel area (TVA) measurements by MRI and histopathology for all coronary artery segments (large graph on top) and for each coronary artery separately (small graphs on bottom). TVA indicates total vessel area; LDA, left anterior descending coronary artery; LCx, left circumflex coronary artery; and RCA, right coronary artery. 


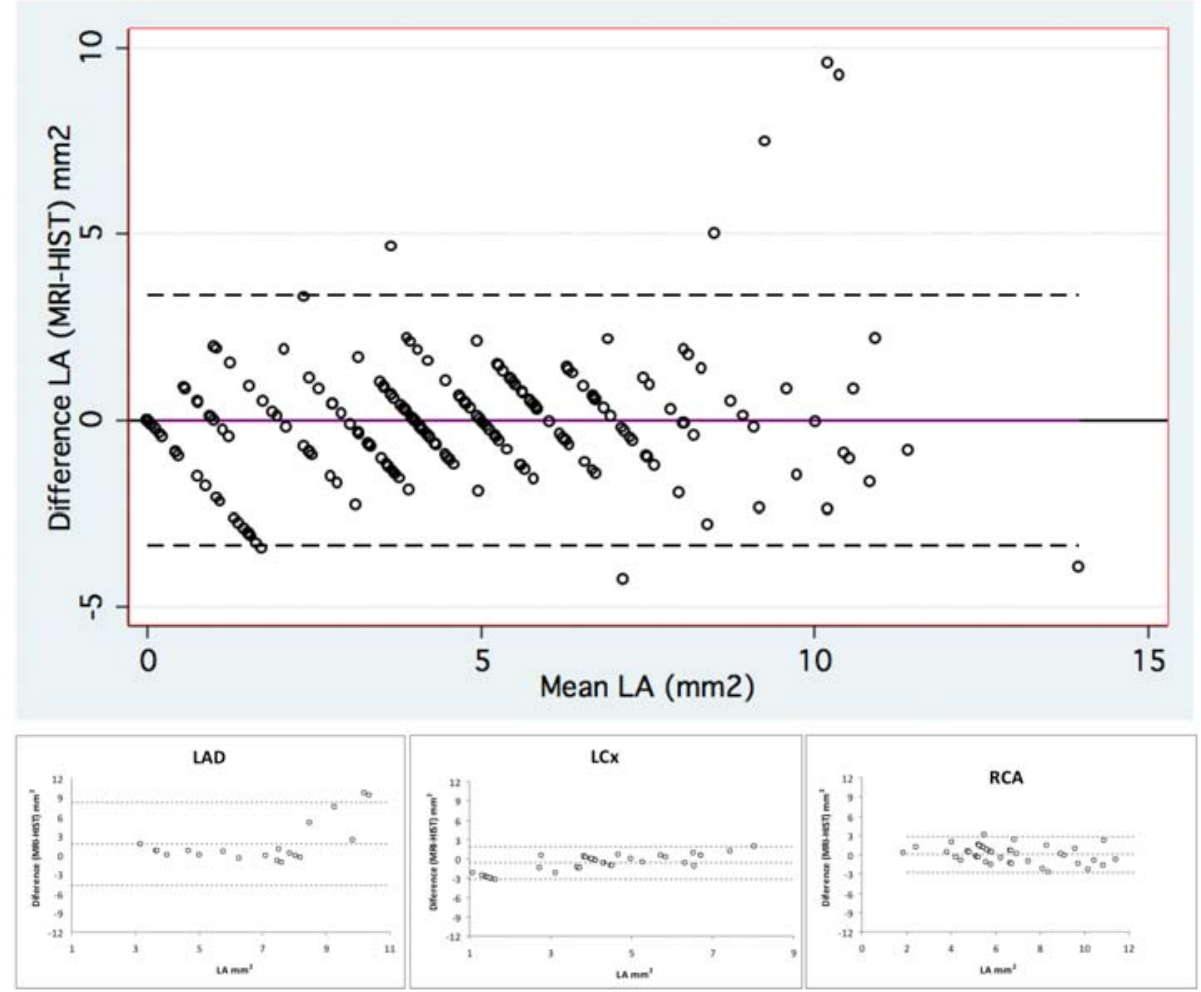

Fig. (3). Bland-Altman graphs illustrating the agreement between lumen area (LA) measurements by MRI and histopathology for all coronary artery segments (large graph on top) and for each coronary artery separately (small graphs on bottom). LA indicates lumen area; LDA, left anterior descending coronary artery; LCx, left circumflex coronary artery; and RCA, right coronary artery.

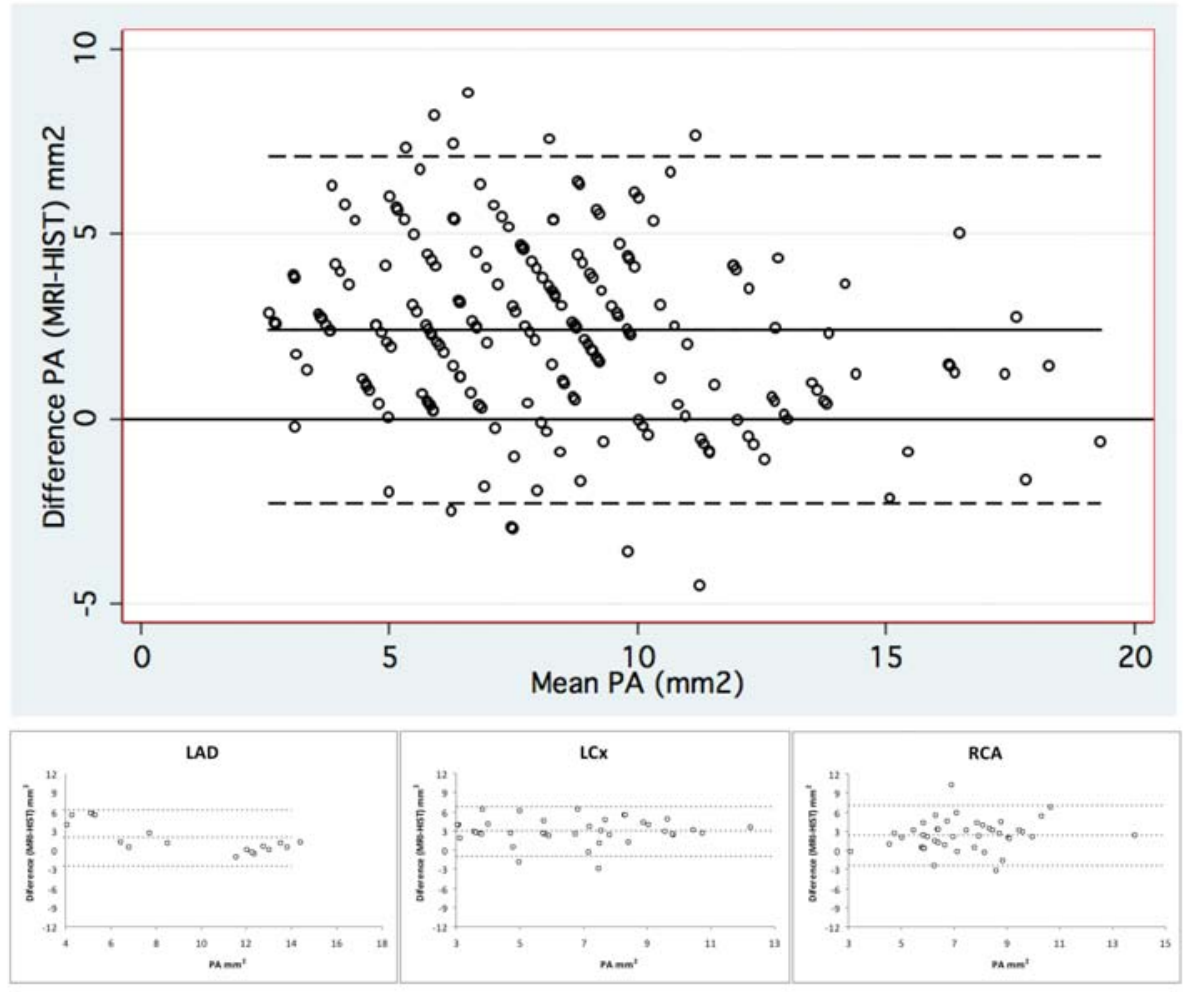

Fig. (4). Bland-Altman graphs illustrating the agreement between plaque area (PA) measurements by MRI and histopathology for all coronary artery segments (large graph on top) and for each coronary artery separately (small graphs on bottom). PA indicates plaque area; LDA, left anterior descending coronary artery; LCx, left circumflex coronary artery; and RCA, right coronary artery. 
Atherosclerosis is the leading cause of morbidity and mortality in industrialized societies, and its incidence is projected to increase in the future [1]. Considering that atherosclerotic disease begins in the vessel wall, the field of cardiovascular imaging is shifting its focus from the assessment of the arterial lumen to imaging of the vessel wall, with the goal of detecting preclinical atherosclerosis. Cardiovascular MRI has been shown to represent a valuable tool for the assessment of various ischemic [16-18] and non-ischemic cardiomyopathies [19-23]. Given its high resolution, 3D capabilities, non-invasive nature, absence of ionizing radiation and the capacity for tissue characterization, MRI is emerging as an important modality for the assessment of atherosclerotic plaque burden in different arterial beds, including the carotid arteries, aorta, and more recently, the coronary arteries [4-6].

Previous studies have evaluated the value of MRI for the assessment of atherosclerotic disease, both ex vivo and in vivo [8-14]. Most of these studies have evaluated atherosclerotic plaques in larger vessels, such as the aortic wall or the carotid artery. Yuan et al. have performed extensive validation work on the carotid plaques [10, 24]. They were the first to investigate the accuracy of arterial wall measurement using carotid high-resolution MRI in patients scheduled for endarterectomy. They performed both in vivo and ex vivo analyses and demonstrated a high degree of agreement of vessel wall area measurements [10]. They concluded that MRI enables accurate quantitative assessment of vessel wall area in atherosclerotic carotid lesions.

There has been also extensive work evaluating the aortic wall. Fayad et al. assessed atherosclerotic plaques in the thoracic aorta both by MRI and by transoesophageal echocardiography [11]. They were able to demonstrate a strong degree of concordance between both methods for plaque composition, mean maximum plaque thickness and overall aortic plaque extent. Steen et al. have also published very interesting studies evaluating the characterization of atherosclerotic disease in the aortic wall $[8,9,25]$. Using an experimental model of combined transoesophageal and surface MRI they managed to generate high quality aortic wall images and achieved a very good degree of reproducibility of aortic wall and atherosclerotic plaque measurements [9]. It is important to recognize that these previous studies have reported values of accuracy and reproducibility of atherosclerosis assessment that are better than the results of the present study. However, these previous studies evaluated much larger and accessible vessels. In contrast, in the present study we evaluated small coronary artery segments in a complex ex-vivo model that allowed us to compare with a specific histopathology protocol that included pressurized lumen fixation as the reference standard.

Indeed, the ultimate goal of non-invasive atherosclerosis assessment is the evaluation and characterization of coronary artery atherosclerotic plaques. Worthley et al. were the first to report on the feasibility of MR imaging and characterizing of atherosclerotic lesions from in situ coronary arteries and aortas in an ex vivo porcine model [26]. They also validated MRI measurements using histopathology as the reference standard. They were able to demonstrate very good agreement between MRI and histopathology measurements. In humans, validation was initially performed ex vivo using histological specimens. In an elegant study, Toussaint et al. used high-field MR spectroscopy to characterize coronary atherosclerotic plaques [27]. Using a combination of T1 weighted and $\mathrm{T} 2 \mathrm{w}$ sequences they were able to perform the in vitro identification of the atheromatous core, collagenous cap, calcifications, media, adventitia and perivascular fat. These previous reports are in agreement with the results of the present study. We were also able to find, using a novel ex vivo coronary imaging, a good agreement between the non-invasive assessment of coronary artery morphology by MRI and the results obtained by the reference standard histopathological analyses.

Autopsy specimens may carry important pathophysiological information on the disease process, particularly, on coronary artery disease, some of them hard to tackle by destructive pathological techniques. Ex vivo studies of human coronary arteries by MRI are not common and techniques developed to optimize high spatial resolution images at the pathological level are needed to further explore this technique. The imaging techniques developed on this study might help researchers to investigate more efficiently coronary artery specimens through plaque tissue characterization and morphological determination of the vessel in a variety of disease processes. Even though this is an experimental ex vivo study, we believe our results might represent an early step in research that could lead to significant clinical implications in the future. As previously mentioned, we were able to demonstrate that MRI is capable of characterizing atherosclerotic plaques in excised coronary artery segments. However, it is much more difficult to acquire high quality images of the coronary arteries in a clinical model, due to the constant movement of the heart. Nevertheless, there have been important technological advances in the field of cardiac MRI over the past 20 years, especially in the last few years, which have witnessed an even more accelerated technological progression. Thus, it is possible that we might be able, in the near future, to replicate the MRI techniques used in the present study in the clinical scenario. Then, we would be able to characterize the atherosclerotic plaques of patients with suspected CAD non-invasively, possibly optimizing the riskstratification of these patients through the identification of vulnerable plaques [28]. Undoubtedly, future studies will be necessary to investigate this possibility.

The present study has several limitations. It is not possible to exclude the possibility that the excision of arteries and fixation with formalin might have had some influence on the measurements. Infact, we believe that morphological changes caused by postmortem shrinkage and fixation techniques might have resulted in overestimation of obstructive lesions and could explain, at least in part, the variance between MRI and histopathology measurements [29]. The ratio diameter of lesion-to-residual lumen can be significantly altered by postmortem changes. For this ratio to change, one would have to assume that following fixation the arterial wall shrank while the atherosclerotic material remained unchanged. On the other hand, atherosclerotic deposits are often eccentric and are not necessarily rigid or calcified. Distension of the artery in vitro by injection of saline solution or soy oil may mask luminal narrowing to some degree, and thus contribute to "underestimation" of lesions. In addition, 
distension of the artery in vitro or in vivo by injection of contrast or solution, or in vivo by the blood pressure itself may mask luminal narrowing to some degree, and thus contribute to underestimation of lesions. Finally, the registration of MR cross-sectional images and the corresponding histopathological slices might not have been sufficiently accurate in some arterial segments. Since only a small degree of misalignment could have a significant influence on the degree of agreement between both methods, we believe this potential limitation might have caused an underestimation of the capacity of MRI to accurately characterize coronary artery morphology when compared to histopathology.

\section{CONCLUSION}

In recent years, MRI has emerged as one of the most promising techniques for the detailed assessment of atherosclerotic disease. In the present study we were able to demonstrate good agreement between coronary artery morphometrical measurements obtained by high-resolution MRI and by histopathology in an ex vivo experimental model. Further studies will be necessary to investigate whether the techniques described in the present study could be used for a more detailed characterization of atherosclerotic disease, including the assessment of the lipid core, the collagenous cap and the degree of positive remodeling.

\section{CONFLICT OF INTEREST}

The authors confirm that this article content has no conflicts of interest.

\section{ACKNOWLEDGEMENTS}

The authors Carlos E. Rochitte, Clerio F. Azevedo, Pedro A. Lemos and Paulo S. Gutierrez were supported by research grants from the National Council for Scientific and Technological Development (CNPq), Brazil.

\section{PATIENT'S CONSENT}

Declared none.

\section{REFERENCES}

[1] Go AS, Mozaffarian D, Roger VL, et al. Heart disease and stroke statistics--2013 update: a report from the American Heart Association. Circulation 2013; 127: e6-245.

[2] Patel MR, Bailey SR, Bonow RO, et al. ACCF/SCAI/AATS/AHA/ASE/ASNC/HFSA/HRS/SCCM/SCCT/ SCMR/STS 2012 appropriate use criteria for diagnostic catheterization: a report of the American College of Cardiology Foundation. Appropriate use criteria task force, Society for Cardiovascular Angiography and Interventions, American Association for Thoracic Surgery, American Heart Association, American Society of Echocardiography, American Society of Nuclear Cardiology, Heart Failure Society of America, Heart Rhythm Society, Society of Critical Care Medicine, Society of Cardiovascular Computed Tomography, Society for Cardiovascular Magnetic Resonance, and Society of Thoracic Surgeons. J Am Coll Cardiol 2012; 59: 19952027.

[3] Van Ditzhuijzen NS, Van Beusekom HM, Ligthart JM, Regar E. Invasive imaging of the coronary atherosclerotic plaque. Minerva Cardioangiol 2012; 60: 305-29.

[4] Bluemke DA, Achenbach S, Budoff M, et al. Non-invasive coronary artery imaging: magnetic resonance angiography and multidetector computed tomography angiography: a scientific statement from the American Heart Association Committee on Cardiovascular Imaging and Intervention of the Council on Cardiovascular Radiology and Intervention, and the Councils on Clinical Cardiology and Cardiovascular Disease in the Young. Circulation 2008; 118: 586-606.

[5] Corti R, Fuster V. Imaging of atherosclerosis: magnetic resonance imaging. Eur Heart J 2011; 32: 1709-19b.

[6] Desai MY, Lima JA. Imaging of atherosclerosis using magnetic resonance: state of the art and future directions. Curr Atheroscler Rep 2006; 8: 131-9.

[7] Corti R, Fayad ZA, Fuster V, et al. Effects of lipid-lowering by simvastatin on human atherosclerotic lesions: a longitudinal study by high-resolution, non-invasive magnetic resonance imaging. Circulation 2001; 104: 249-52.

[8] Steen H, Lima JA, Chatterjee S, et al. High-resolution threedimensional aortic magnetic resonance angiography and quantitative vessel wall characterization of different atherosclerotic stages in a rabbit model. Invest Radiol 2007; 42: 614-21.

[9] Steen H, Warren WP, Desai M, et al. Combined transesophageal and surface MRI provides optimal imaging in aortic atherosclerosis. J Cardiovasc Magn Reson 2004; 6: 909-16.

[10] Yuan C, Beach KW, Smith LH Jr, Hatsukami TS. Measurement of atherosclerotic carotid plaque size in vivo using high-resolution magnetic resonance imaging. Circulation 1998; 98: 2666-71.

[11] Fayad ZA, Nahar T, Fallon JT, et al. In vivo magnetic resonance evaluation of atherosclerotic plaques in the human thoracic aorta: a comparison with transesophageal echocardiography. Circulation 2000; 101: 2503-9.

[12] Botnar RM, Stuber M, Kissinger KV, Kim WY, Spuentrup E, Manning WJ. Non-invasive coronary vessel wall and plaque imaging with magnetic resonance imaging. Circulation 2000; 102: 25827.

[13] Fayad ZA, Fuster V, Fallon JT, et al. Non-invasive in vivo human coronary artery lumen and wall imaging using black-blood magnetic resonance imaging. Circulation 2000; 102: 506-10.

[14] Stuber M, Botnar RM, Danias PG, Kissinger KV, Manning WJ. Submillimeter three-dimensional coronary MR angiography with real-time navigator correction: comparison of navigator locations. Radiology 1999; 212: 579-87.

[15] Bland JM, Altman DG. Statistical methods for assessing agreement between two methods of clinical measurement. Lancet 1986;1: 307-10.

[16] Azevedo Filho CFd, Hadlich M, Petriz JLF, Mendonça LA, Moll Filho JN, Rochitte CE. Quantification of left ventricular infarcted mass on cardiac magnetic resonance imaging: comparison between planimetry and the semiquantitative visual scoring method. Arq Bras Cardiol 2004; 83: 118-24.

[17] Kim RJ, Wu E, Rafael A, et al. The use of contrast-enhanced magnetic resonance imaging to identify reversible myocardial dysfunction. N Engl J Med 2000; 343: 1445-53.

[18] Rettmann DW, Saranathan M, Wu KC, Azevedo CF, Bluemke DA, Foo TK. High temporal resolution breathheld 3D FIESTA CINE imaging: validation of ventricular function in patients with chronic myocardial infarction. J Magn Reson Imaging 2007; 25: 1141-6.

[19] Azevedo CF, Nigri M, Higuchi ML, et al. Prognostic significance of myocardial fibrosis quantification by histopathology and magnetic resonance imaging in patients with severe aortic valve disease. J Am Coll Cardiol 2010; 56: 278-87.

[20] Nigri M, Azevedo CF, Rochitte CE, et al. Contrast-enhanced magnetic resonance imaging identifies focal regions of intramyocardial fibrosis in patients with severe aortic valve disease: Correlation with quantitative histopathology. Am Heart J 2009; 157: 361-8.

[21] O'Hanlon R, Grasso A, Roughton M, et al. Prognostic significance of myocardial fibrosis in hypertrophic cardiomyopathy. J Am Coll Cardiol 2010; 56: 867-74.

[22] Rochitte CE, Oliveira PF, Andrade JM, et al. Myocardial delayed enhancement by magnetic resonance imaging in patients with Chagas' disease: a marker of disease severity. J Am Coll Cardiol 2005; 46: $1553-8$.

[23] Wu KC, Weiss RG, Thiemann DR, et al. Late gadolinium enhancement by cardiovascular magnetic resonance heralds an adverse prognosis in nonischemic cardiomyopathy. J Am Coll Cardiol 2008; 51: 2414-21.

[24] Yuan C, Mitsumori LM, Ferguson MS, et al. In vivo accuracy of multispectral magnetic resonance imaging for identifying lipid-rich 
necrotic cores and intraplaque hemorrhage in advanced human carotid plaques. Circulation 2001; 104: 2051-6.

[25] Lima JA, Desai MY, Steen H, Warren WP, Gautam S, Lai S. Statin-induced cholesterol lowering and plaque regression after 6 months of magnetic resonance imaging-monitored therapy. Circulation 2004; 110: 2336-41.

[26] Worthley SG, Helft G, Fuster V, et al. High resolution ex vivo magnetic resonance imaging of in situ coronary and aortic atherosclerotic plaque in a porcine model. Atherosclerosis 2000; 150: 321-9.
[27] Toussaint JF, Southern JF, Fuster V, Kantor HL. T2-weighted contrast for NMR characterization of human atherosclerosis. Arterioscler Thromb Vasc Biol 1995; 15: 1533-42.

[28] Stone GW, Maehara A, Lansky AJ, et al. A prospective naturalhistory study of coronary atherosclerosis. N Engl J Med 2011; 364 226-35.

[29] Vlodaver Z, Frech R, Van Tassel RA, Edwards JE. Correlation of the antemortem coronary arteriogram and the postmortem specimen. Circulation 1973; 47: 162-9.

Received: December 20, 2013

Revised: January 31, 2014

Accepted: February 04, 2014

(c) Gomes et al.; Licensee Bentham Open.

This is an open access article licensed under the terms of the Creative Commons Attribution Non-Commercial License (http://creativecommons.org/licenses/by-nc/3.0/) which permits unrestricted, non-commercial use, distribution and reproduction in any medium, provided the work is properly cited. 